\title{
The joy of parenting: infant sleep intervention to improve maternal emotional well-being and infant sleep
}

\author{
Brian Symon ${ }^{1,2}$, MD, MBBs, Georgina E Crichton ${ }^{2,3}, \mathrm{PhD}$
}

INTRODUCTION This study aimed to examine how improving infant sleep impacted the emotional well-being of mothers. METHODS The participants were 80 mothers of infants aged 6-12 months; they attended a primary care medical clinic in Adelaide, Australia, for assistance with infant sleep problems. Behavioural intervention consisted of a 45-minute consultation, where verbal and written information describing sleep physiology and strategies to improve infant sleep was provided. Mothers were followed up 2-6 weeks later. Mothers rated their confidence (C), pleasure (P) and frustration (F) on a scale from 0 to 10, and completed the Depression Anxiety Stress Scale 21 at each consultation. The number of night-time awakenings and time taken to see an improvement in infant sleep were also reported.

RESULTS There was a significant increase in the $C$ and $P$ scores, and a significant decrease in the $F$ scores $(a l l p<0.001)$. The mean total CPF score increased significantly from 14 to 25 (maximum score $=30$ ). There was also a significant decrease in depression, anxiety and stress in the mothers (all $p<0.001$ ). The mean number of maximum night awakenings also decreased significantly, from 4.9 to $0.5(p<0.001)$. The mean time taken to see improved infant sleep, as reported by the mothers, was 2.8 nights.

CONCLUSION A single consultation using a behavioural strategy to improve infant sleep was effective in improving infant sleep and in increasing maternal emotional well-being. In particular, the scores for 'pleasure in being a mother' increased dramatically.

Keywords: behaviour modification, infant sleep, maternal well-being, postnatal depression

\section{INTRODUCTION}

Sleep problems in infancy are reported by up to $46 \%$ of parents. ${ }^{(1,2)}$ Sleep problems can result in negative consequences for both the mother and child. Infant sleep problems are frequently associated with maternal depression and anxiety, in addition to poor maternal physical health and reduced quality of life. ${ }^{(2-6)}$ Postnatal depression can have serious and long-term detrimental effects on the family's well-being and stability, the mother-child relationship, and the cognitive and emotional development of the child..$^{(7-9)}$ Disrupted and short sleep duration in early infancy can also have direct and negative impacts on the child's behavioural, cognitive and physical development; it can also increase the risk of being overweight and having further sleep problems in childhood..$^{10-12}$

Behaviours that may be characterised as sleep problems by parents include frequent night-time waking, needing to be nursed to sleep, requiring prolonged periods to fall asleep, and co-sleeping. Randomised trials have shown that behavioural interventions are able to effectively reduce sleep problems in children and associated maternal depression; the interventions were also shown to improve other domains of parental wellbeing. ${ }^{(5,13-18)}$ These interventions, which involve discussions on infant sleep and the provision of advice on sleep improvement strategies, have been shown to be effective in children aged six weeks to two years. ${ }^{(13,18)}$ In these studies, the common information provided was advice regarding the development of positive routines for feeding and sleeping, particularly at bedtime, to best facilitate both. A key component was the recommendation to let the child achieve sleep with minimal parental interference, thus teaching the child to learn self-settling skills. Parents are advised to 'check' on their infant while the infant is asleep, but to refrain from physical contact with the infant while he/she is crying in the night. Parents are taught that they are viewed as 'reward objects' to their infants, and therefore, attending to a child who is crying or displaying other inappropriate behaviour only serves to reward and reinforce such behaviour.

In our previous randomised controlled trial, ${ }^{(18)}$ families with newborn infants were recruited at birth and randomly assigned to either an intervention group or a control group. The control received routine care, while the intervention group received a single 45-minute consultation with a trained research nurse when the infant was aged between two and three weeks. The consultation session comprised a discussion on normal sleep patterns and the use of behavioural techniques to develop 'independent sleep skills' in the infant. At both six and 12 weeks of age, the infants in the intervention group showed significant sleep improvement, including higher mean hours of day, night and total sleep, without an increase in infant crying. By 12 weeks of age, the infants in the intervention group averaged 8.4 hours more sleep per week than the infants in the control group.

We also demonstrated that applying the same behavioural intervention in infants aged 6-12 months with established infant sleep problems is beneficial for infant sleep. ${ }^{(19)}$ Within three weeks of the intervention, the mean number of nighttime awakenings significantly decreased from 4.9 to 0.5 (mean difference 4.4). Furthermore, maternal depression, anxiety and stress decreased significantly (all $p<0.01$ ); the

${ }^{1}$ University of Adelaide, ${ }^{2}$ Kensington Park Medical Practice, Kensington Park, ${ }^{3}$ Alliance for Research in Exercise, Nutrition and Activity (ARENA), Sansom Institute for Health Research, University of South Australia, Adelaide, Australia

Correspondence: Dr Georgina Crichton, Kensington Park Medical Practice, 84 Shipsters Road, Kensington Park, South Australia 5068, Australia. georgina.crichton@unisa.edu.au 
overall proportion of mothers assessed as having any degree of depression decreased by $85 \%$.

The aforementioned findings are supported by the Infant Sleep Study, a large community-based, randomised controlled trial of a brief sleep intervention conducted in eight- to ten-month-old infants with identified sleep problems, with recurrent follow-up periods. ${ }^{(5)}$ Following a similar behavioural intervention, significant improvements in infant sleep were observed at ten and 12 months; these improvements were maintained at two years and significant improvements in maternal mental health were also noted..$^{(5,13,20)}$

The aim of the present study was to measure additional outcomes linked to the behavioural intervention, which were not assessed in other studies. While many new mothers may not meet the clinical definition of having postnatal depression, some experience emotional problems that may still negatively impact their well-being. ${ }^{(2,21)}$ High levels of frustration and/or low levels of confidence and pleasure as a parent may negatively impact the mother, her infant and her family unit. Other than that, few studies have collected information or reported on the time taken to achieve improvement in infant sleep following behavioural interventions.

Parents may find it difficult to listen to the cries of their infant. If trials demonstrate rapid sleep improvement after such behavioural interventions, more families may be willing to implement the behavioural interventions. Herein, we report the secondary outcomes (i.e. self-rated maternal confidence, pleasure and frustration; and time taken to observe an improvement in infant sleep) of an original study ${ }^{(19)}$ that was conducted in selfselected families that experienced sleep problems in infants aged between six and 12 months. In summary, the present study aimed to extend the information that is available in the current literature by: (a) evaluating other maternal indicators of psychological wellbeing that are associated with motherhood; and (b) measuring the time taken to achieve improvement in infant sleep following behavioural intervention.

\section{METHODS}

Consecutive mothers of young babies who presented to a metropolitan general medical practice in Adelaide, South Australia, Australia, requesting assistance with infant sleep problems were invited to participate in a study of maternal health and well-being. The details of the study design and sampling procedure have been previously reported. ${ }^{(19)}$ The medical practice specialises in the management of sleep and feeding problems in babies and children. Mother-child pairs were excluded if the child was aged $<6$ months or $>12$ months, or if the mother-child pair had previously attended the clinic. All consultations were conducted in English and all the mothers included in the present study conversed fluently in the English language, although the inability to speak English was not a reason for exclusion. A total of 103 mothers, aged 20-45 years, were invited to participate in this study, and 101 of them provided written consent and completed the baseline clinical interview. Of the 101 mothers, 80 (79.2\%) completed at least one component of the follow-up. Ethics approval was granted by the University of Adelaide Ethics Committee.
The behavioural sleep intervention comprised a 45-minute, one-on-one consultation with an experienced general practitioner (GP) who had expertise in infant sleep, or a registered nurse who was trained and supervised by a GP to provide the same intervention. Participants seen by the nurse were reviewed by the GP. This intervention was previously described and included a discussion on normal sleep patterns in newborn infants. ${ }^{(18,19)}$ In summary, the following key information was given to the mothers during the consultation: (a) human sleep is cyclical; (b) a return to sleep from an awakening is in part driven by external cues of sleep; (c) cues of sleep are learned; (d) sleep is usefully regarded as a learned skill; (e) fatigue interferes with the performance of learned skills (i.e. fatigue impairs performing the skills of achieving and maintaining sleep); and ( $f$ ) the cues of sleep that are parent-independent are the most useful. The GP or nurse would emphasise to the mothers that sleep achievement and maintenance are learned skills, and that there is a need to avoid cues of sleep that include parental care, as this can lead to repeated cycles of 'requesting' parental support within a block of sleep. Implementing bedtime routines and putting the infant to sleep while awake, without parental intervention, in a safe environment are key points in encouraging the child to self-settle and develop efficient sleep habits. On top of the 45-minute consultation, the participants were provided a written copy of the information given during the consultation. They were also encouraged to access a website (http://www.silentnights.org/), where a range of supporting material was available.

The Depression Anxiety Stress Scale 21 (DASS 21) is a validated 21 -item, self-report measure designed to assess anxiety, depression and stress. ${ }^{(22)}$ It was completed by all the participants prior to their initial consultation with the clinician. There are three scales of seven items in the DASS 21 and each item is scored on a four-point scale, where $0=$ 'did not apply to me at all' and 4 = 'applied to me very much, or most of the time'. The scores for depression, anxiety and stress were calculated by summing up the scores of the relevant items and multiplying the sum by two (to enable comparison with full DASS scores and clinical cut-offs). A severity rating, ranging from 'normal' to 'extremely severe', was calculated for each component. Numerous studies have demonstrated the excellent reliability, and convergent and discriminant validity of the DASS. ${ }^{(23-25)}$ Follow-up consultation took place at the clinic 2-6 weeks later (the median time was 25 days). At follow-up consultation, the participants $(n=52)$ were asked to complete the DASS 21 again, prior to their consultation with the clinician. The DASS 21 was completed by phone interview for those who were unable to return to the clinic $(n=28)$ for the follow-up consultation. The phone interviews were conducted by a trained researcher, who delivered the DASS 21 in a standardised way. All interviews were conducted in English, as there were no non-English-speaking participants. Overall, the 80 mothers completed the DASS 21 twice - once prior to initial consultation and once prior to follow-up consultation.

At the baseline clinical interview with the clinician, each mother was asked to rate her feelings of confidence $(C)$, pleasure $(\mathrm{P})$ and frustration $(\mathrm{F})$ on a Likert-type scale, which 
Table I. Comparison of the baseline demographic characteristics of those who completed at least one follow-up component of the study and those who did not.

\begin{tabular}{|c|c|c|c|c|}
\hline \multirow[t]{2}{*}{ Variable at baseline } & \multicolumn{3}{|c|}{ Mean \pm standard deviation } & \multirow[t]{2}{*}{ p-value } \\
\hline & Completed $(n=80)$ & Withdrew $(n=21)$ & Total $(n=101)$ & \\
\hline Maternal age (yr) & $32.5 \pm 5.0$ & $32.0 \pm 4.7$ & $32.4 \pm 4.9$ & 0.72 \\
\hline Child's age (mth) & $8.5 \pm 1.7$ & $8.4 \pm 2.3$ & $8.4 \pm 1.8$ & 0.83 \\
\hline Min no. of night awakenings & $3.0 \pm 2.0$ & $3.6 \pm 2.2$ & $3.2 \pm 2.1$ & 0.27 \\
\hline Max no. of night awakenings & $4.4 \pm 3.3$ & $5.2 \pm 4.0$ & $4.6 \pm 3.4$ & 0.37 \\
\hline P score* & $6.0 \pm 2.9$ & $6.0 \pm 3.4$ & $6.0 \pm 3.0$ & 0.98 \\
\hline F score* & $7.6 \pm 2.2$ & $6.4 \pm 2.6$ & $7.3 \pm 2.4$ & 0.06 \\
\hline Total CPF score ${ }^{+}$ & $13.3 \pm 6.0$ & $14.0 \pm 6.7$ & $13.5 \pm 6.1$ & 0.63 \\
\hline DASS 21 score & $29.1 \pm 21.1$ & $27.3 \pm 20.0$ & $28.7 \pm 20.8$ & 0.74 \\
\hline
\end{tabular}

Independent sample $t$-tests were used to calculate the $\mathrm{p}$-values for the difference between means. * Confidence $(\mathrm{C})$, pleasure $(\mathrm{P})$ and frustration $(\mathrm{F})$ were measured on a Likert-type scale that ranged from 0 to $10 .{ }^{\dagger}$ Total CPF score was calculated using the following formula: $\mathrm{C}+\mathrm{P}-\mathrm{F}+10$; the resulting value could range from 0 to 30. DASS 21: 21-item Depression Anxiety Stress Scale

Table II. Maternal confidence (C), pleasure (P) and frustration (F) scores at baseline and follow-up, and the time taken to see an improvement in infant sleep.

\begin{tabular}{|c|c|c|c|c|c|}
\hline \multirow[t]{2}{*}{ Variable } & \multirow[t]{2}{*}{ No. of mothers } & \multicolumn{2}{|c|}{ Mean \pm SD } & \multirow[t]{2}{*}{ Mean change $(95 \% \mathrm{CI})$} & \multirow[t]{2}{*}{ p-value } \\
\hline & & At baseline & At follow-up & & \\
\hline C score* & 49 & $5.2 \pm 2.3$ & $8.4 \pm 1.7$ & 3.2 (2.4 to 4.0$)$ & $<0.001$ \\
\hline P score* & 49 & $6.1 \pm 2.8$ & $9.2 \pm 1.3$ & 3.1 (2.4 to 4.0$)$ & $<0.001$ \\
\hline F score $^{*}$ & 49 & $7.4 \pm 2.5$ & $2.5 \pm 2.3$ & $-5.0(-5.8$ to -4.0$)$ & $<0.001$ \\
\hline Total CPF score ${ }^{\dagger}$ & 49 & $13.9 \pm 5.9$ & $24.6 \pm 5.0$ & 10.7 (8.6 to 12.8$)$ & $<0.001$ \\
\hline
\end{tabular}

Paired sample $t$-tests were used to calculate the $\mathrm{p}$-values for the difference between the two time points. ${ }^{*} \mathrm{C}, \mathrm{P}$ and $\mathrm{F}$ were measured on a Likert-type scale that ranged from 0 to 10 . †Total CPF score was calculated using the following formula: $C+P-F+10$; the resulting value could range from 0 to 30 . Cl: confidence interval; SD: standard deviation

ranged from 0 (i.e. no confidence/no pleasure/no frustration) to 10 (i.e. very confident/maximal pleasure/very frustrated). On the $\mathrm{C}$ and $\mathrm{P}$ scales, low scores were representative of negative feelings and high scores were representative of positive feelings. On the $\mathrm{F}$ scale, however, low scores were representative of positive feelings, while high scores were representative of negative feelings. A score of 5 was typically perceived as neutral (i.e. there were neither positive nor negative feelings). The total CPF score was calculated using the following formula: $\mathrm{C}+\mathrm{P}-$ $\mathrm{F}+10$. Higher scores indicated greater feelings of well-being (i.e. greater confidence and pleasure, and less frustration). The $\mathrm{C}, \mathrm{P}$ and $\mathrm{F}$ scores at follow-up were obtained from the mothers who returned to the practice for a second clinical interview with the clinician $(n=49)$.

The number of usual night-time awakenings (minimum and maximum number), as reported by the mothers, was collected at baseline and follow-up. At follow-up, conducted the practice or by telephone, the mothers $(\mathrm{n}=74)$ were asked how many nights it took for any improvement in infant sleep to be observed. A response of ' 0 ' indicated that improvement was observed on the first night.

Independent sample $t$-tests were used to compare baseline demographic characteristics and DASS 21 scores between those who completed the DASS component of the study at follow-up ( $n=80$ ) and those who did not complete at least one component of the follow-up $(n=21)$. Paired $t$-tests were used to determine whether there was any significant change in the $\mathrm{C}$, $\mathrm{P}, \mathrm{F}$ and total CPF scores of each participant between the two time points (i.e. baseline and follow-up). All statistical analyses were conducted using PASW Statistics for Windows version 18.0 (SPSS Inc, Chicago, IL, USA). A p-value $<0.05$ was considered significant for the primary outcomes measured.

\section{RESULTS}

At baseline, there were no significant differences in the mother's age, the child's age, the mother's C, P and F scores, and the number of night awakenings between those who completed the study ( $n=80)$ and those who did not $(n=21)$ (Table I). The mothers came from a wide variety of socioeconomic backgrounds, as demonstrated by their area of residence (data not shown).

The mean number of nights taken to see an improvement in infant sleep was $2.8 \pm 2.9$ nights (Table II). As reported previously, ${ }^{(19)}$ the minimum number of night-time awakenings significantly decreased from $3.3 \pm 2.2$ nights to $0.4 \pm 0.8$ nights, and the maximum number of night-time awakenings significantly decreased from $4.9 \pm 3.6$ nights to $0.5 \pm 0.8$ nights (both $\mathrm{p}<0.0001$ ). In the present study, $2 \%$ of the mothers reported no night-time awakenings at baseline; this percentage increased to $69 \%$ at follow-up. ${ }^{(19)}$ At baseline, $23 \%$ of the mothers experienced five or more night-time awakenings, while at follow-up, this percentage was reduced to $0 \%$. 
49 mothers returned to the practice for the second clinical interview; it was during this interview that the follow-up C, P and $\mathrm{F}$ scores were obtained. Table II shows the mothers' mean C, P, F and total CPF scores, at baseline and at follow-up. The mean $\mathrm{C}$ score and mean $\mathrm{P}$ score increased significantly following the sleep intervention (mean increase of three points for both, $p<0.001)$. There was also a significant decrease in the mean $\mathrm{F}$ score following the sleep intervention (mean decrease of five points, $p<0.001)$. A corresponding increase was observed in the mean total CPF score following the sleep intervention (mean increase of 11 points, $p<0.001$ ). An intriguing observation of this study was that a large proportion of the mothers reporting on their pleasure in being a parent at the second visit ignored the $\mathrm{P}$ scale. That is, rather than giving a score out of ten, as requested, they ignored that scale and gave a non-reportable result such as ' $100 / 10^{\prime}$ or 'infinity/10'. This is a unique experience to the study team and it appears to reflect a profound increase in the 'joy of parenting' experienced by mothers once the infant's sleep patterns have been corrected. In fact, some mothers reported a renewed interest in considering another baby.

As previously reported, ${ }^{(19)}$ there were significant reductions in maternal depression, anxiety and stress after the intervention (all $p<0.001$ ); there was also a mean reduction of $48.8 \%$ in the total DASS 21 scores. The proportion of mothers assessed as having any degree of depression decreased by $85 \%$, from $26 / 80(32.5 \%)$ to $4 / 80(5.0 \%) .{ }^{(19)}$

\section{DISCUSSION}

We have demonstrated that a simple behavioural intervention conducted in a single clinic visit can significantly increase maternal self-rated confidence and pleasure in parenting, as well as decrease maternal self-rated frustration in parenting. The results of the present study also showed that improvements in infant sleep patterns can be observed in a short period of time. In this study, the mean time taken to see improved infant sleep was less than three nights. This adds to our previous findings, ${ }^{(19)}$ which showed that advice on healthy sleep habits provided at clinic consultations can be effective in reducing both infant night-time awakenings and maternal depression, anxiety and stress.

Despite the available evidence supporting the efficacy of this approach for both the mother and child, ${ }^{(5,17,19)}$ many paediatricians and infant/child services staff are not in favour of advocating such techniques due to concerns that there may be long-term harm in terms of parent-child bonding and the child's emotional development. ${ }^{(26)}$ However, follow-up studies are now providing evidence that such behavioural sleep interventions have no longlasting negative effects on a child's behaviour, emotional conduct and sleep pattern, the parent-child closeness/relationship, and parental psychological well-being. ${ }^{(5,6,13,15,20,27)}$

Poor infant sleep habits may have a particularly adverse impact on mothers who are already experiencing some degree of postnatal depression or feelings of low self-confidence during early motherhood. The provision of a simple, consultation-based strategy has the potential to benefit such women. The intervention can be readily taught to healthcare professionals in a primary care setting, and from a public health perspective, it is an extremely cost- and time-effective means of improving infant sleep and maternal well-being.

The present study was not without limitations. The participants of the study were mothers presenting to a local medical practitioner for assistance with infant sleep problems. In other words, these mothers had identified a problem and wanted to seek help. The mothers were representative of a wide range of socioeconomic strata; however, women who were aged $<20$ years and from a non-English-speaking background were likely to have been unrepresented in the study. Also, the study did not examine the long-term impact of behavioural intervention on infant sleep performance and maternal wellbeing. Unfortunately, the mothers who completed the followup DASS 21 over the phone did not complete the C, P and F ratings. However, no significant differences in the baseline scores of these measures were observed between the mothers who completed the follow-up C, P and F ratings and the mothers who did not. Despite this, reporting bias cannot be ruled out. We acknowledge that there have been no formal tests on the reliability or validity of the $\mathrm{C}, \mathrm{P}$ and $\mathrm{F}$ Likert-type scales that were used in this study, as these scales were newly developed by the medical practitioner. Other than that, no open-ended questions were used in conjunction with these scales. The use of open-ended questions may have enabled more information about parental attitudes and perceptions to be captured.

The literature lacks an accepted definition of what constitutes a sleep problem. Parental report of the number of night-time awakenings ascertained that the infants did have interrupted sleep. In fact, as many as 20 night-time awakenings were reported at baseline by one mother. Although it is logical and intuitive that this would have had a negative impact on both the infant and other family members, we are unable to make conclusions regarding the direction of causality between infant sleep problems and maternal psychological health. The intervention employed in the present study aimed to improve infant sleep behaviour and, if successful, benefit maternal well-being. We have shown that the intervention is effective in achieving rapid improvements in infant sleep and substantial improvements in overall maternal well-being. While we cannot specify whether the mothers who did not complete the second clinical interview had the same improvements in confidence, pleasure and frustration as those who did, we speculate that similar benefits would have been observed, as there was no significant difference in the followup DASS 21 scores of those who completed the DASS 21 by telephone and those who completed the DASS 21 at the clinic (the scores improved significantly for both groups). However, as we did not have a distinct control group, we are not able to make conclusions regarding cause and effect.

There are a number of factors that may have contributed to the success or failure of the intervention, and these should be assessed in future studies. Important considerations include stressors, such as non-supportive family members, altered living conditions, employment stress, single parenting, family illness and personal tragedy. The presence of such stressors may contribute 
to a lack of improvement in infant sleep performance and/or maternal mental health.

In conclusion, we have demonstrated that the behavioural intervention used in the present study resulted in a clear and quick improvement in infant sleep, and substantial benefits to the psychological well-being of mothers. The intervention is effective and timely, and the implementation cost is minimal. Managing infant sleep problems with behavioural modification techniques may be effective in rapidly improving infant sleep, improving feelings of confidence and pleasure in young mothers, and reducing feelings of frustration in young mothers. Overall, in the present study, a profound increase in maternal pleasure in parenting was observed after the use of the behavioural intervention.

\section{ACKNOWLEDGEMENT}

The authors wish to thank Mr Michael Bammann for his assistance with the data collection.

\section{REFERENCES}

1. Armstrong KL, Quinn RA, Dadds MR. The sleep patterns of normal children. Med J Aust 1994; 161:202-6.

2. Hiscock $\mathrm{H}$, Wake $\mathrm{M}$. Infant sleep problems and postnatal depression: a community-based study. Pediatrics 2001; 107:1317-22.

3. Armstrong KL, Van Haeringen AR, Dadds MR, Cash R. Sleep deprivation or postnatal depression in later infancy: separating the chicken from the egg. J Paediatr Child Health 1998; 34:260-2.

4. Bayer JK, Hiscock H, Hampton A, Wake M. Sleep problems in young infants and maternal mental and physical health. J Paediatr Child Health 2007; 43:66-73.

5. Hiscock H, Bayer J, Gold L, et al. Improving infant sleep and maternal mental health: a cluster randomised trial. Arch Dis Child 2007; 92:952-8.

6. Lam P, Hiscock H, Wake M. Outcomes of infant sleep problems: a longitudinal study of sleep, behavior, and maternal well-being. Pediatrics 2003; 111:e203-7.

7. Beck CT. The effects of postpartum depression on child development: a meta-analysis. Arch Psychiatr Nurs 1998; 12:12-20.

8. Cogill SR, Caplan HL, Alexandra H, Robson KM, Kumar R. Impact of maternal postnatal depression on cognitive development of young children. Br Med J (Clin Res Ed) 1986; 292:1165-7.

9. Murray L, Cooper PJ. The impact of postpartum depression on child development. Int Rev Psychiatry 1996; 8:55-63.

10. Taveras EM, Rifas-Shiman SL, Oken E, Gunderson EP, Gillman MW. Short sleep duration in infancy and risk of childhood overweight. Arch Pediatr Adolesc Med 2008; 162:305-11.

11. Touchette E, Petit D, Tremblay RE, Montplaisir JY. Risk factors and consequences of early childhood dyssomnias: New perspectives. Sleep Med Rev 2009; 13:355-61.

12. Zuckerman B, Stevenson J, Bailey V. Sleep problems in early childhood: continuities, predictive factors, and behavioral correlates. Pediatrics 1987; 80:664-71.

13. Hiscock H, Bayer JK, Hampton A, Ukoumunne OC, Wake M. Long-term mother and child mental health effects of a population-based infant sleep intervention: cluster-randomized, controlled trial. Pediatrics 2008; 122:e621-7.

14. Hiscock H, Wake M. Randomised controlled trial of behavioural infant sleep intervention to improve infant sleep and maternal mood. BMJ 2002; 324:1062-5

15. Mindell JA, Du Mond CE, Sadeh A, et al. Long-term efficacy of an internetbased intervention for infant and toddler sleep disturbances: one year follow-up. J Clin Sleep Med 2011; 7:507-11.

16. Mindell JA, Du Mond CE, Sadeh A, et al. Efficacy of an internet-based intervention for infant and toddler sleep disturbances. Sleep 2011; 34:451-8.

17. St James-Roberts I, Sleep J, Morris S, Owen C, Gillham P. Use of a behavioural programme in the first 3 months to prevent infant crying and sleeping problems. J Paediatr Child Health 2001; 37:289-97.

18. Symon BG, Marley JE, Martin AJ, Norman ER. Effect of a consultation teaching behaviour modification on sleep performance in infants: a randomised controlled trial. Med J Aust 2005; 182:215-8.

19. Symon B, Bammann M, Crichton G, Lowings C, Tucsok J. Reducing postnatal depression, anxiety and stress using an infant sleep intervention. BMJ Open 2012; 2.

20. Price AM, Wake M, Ukoumunne OC, Hiscock H. Five-year follow-up of harms and benefits of behavioral infant sleep intervention: randomized trial. Pediatrics 2012; 130:643-51.

21. Brown S, Lumley J. Maternal health after childbirth: results of an Australian population based survey. Br J Obstet Gynaecol 1998; 105:156-61.

22. Lovibond SH, Lovibond PF. Manual for the Depression Anxiety Stress Scales. 2nd ed. Sydney: Psychology Foundation; 1995.

23. Akin A, Cetin B. The Depression Anxiety and Stress Scale (DASS): the study of validity and reliability. Ed Sci Theory Prac 2007; 7:260-8.

24. Crawford JR, Henry JD. The Depression Anxiety Stress Scales (DASS): normative data and latent structure in a large non-clinical sample. $\mathrm{Br}$ J Clin Psychol 2003; 42(Pt 2):111-31.

25. Lovibond PF, Lovibond $\mathrm{SH}$. The structure of negative emotional states: comparison of the Depression Anxiety Stress Scales (DASS) with the Beck Depression and Anxiety Inventories. Behav Res Ther 1995; 33:335-43.

26. Blunden SL, Thompson KR, Dawson D. Behavioural sleep treatments and night time crying in infants: challenging the status quo. Sleep Med Rev $2011 ; 15: 327-34$.

27. Mindell JA, Kuhn B, Lewin DS, Meltzer LJ, Sadeh A; American Academy of Sleep Medicine. Behavioral treatment of bedtime problems and night wakings in infants and young children. Sleep 2006; 29:1263-76. 